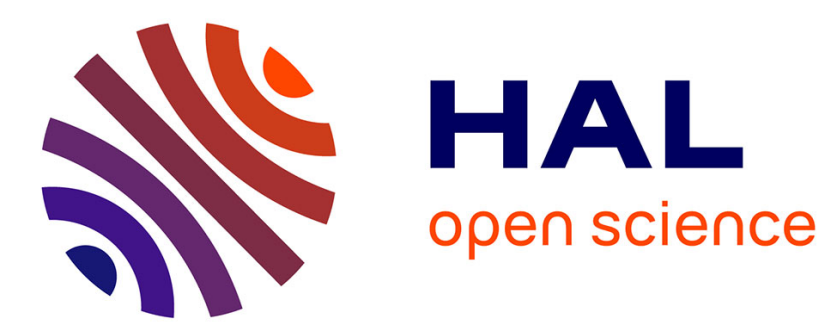

\title{
The Doniach diagram and hydrogenation of the ternary compounds CePdIn and CePdSn
}

\author{
Bernard Chevalier, Alain Wattiaux, Jean-Louis Bobet
}

\section{To cite this version:}

Bernard Chevalier, Alain Wattiaux, Jean-Louis Bobet. The Doniach diagram and hydrogenation of the ternary compounds CePdIn and CePdSn. Journal of Physics: Condensed Matter, 2006, 18 (5), pp.1743-1755. 10.1088/0953-8984/18/5/026 . hal-00018443

\section{HAL Id: hal-00018443 \\ https://hal.science/hal-00018443}

Submitted on 3 Feb 2006

HAL is a multi-disciplinary open access archive for the deposit and dissemination of scientific research documents, whether they are published or not. The documents may come from teaching and research institutions in France or abroad, or from public or private research centers.
L'archive ouverte pluridisciplinaire $\mathbf{H A L}$, est destinée au dépôt et à la diffusion de documents scientifiques de niveau recherche, publiés ou non, émanant des établissements d'enseignement et de recherche français ou étrangers, des laboratoires publics ou privés. 


\title{
Doniach diagram and hydrogenation of the ternary compounds CePdIn and CePdSn
}

\author{
B. Chevalier ${ }^{*}$, A. Wattiaux, J.-L. Bobet \\ Institut de Chimie de la Matière Condensée de Bordeaux (ICMCB), CNRS [UPR 9048], \\ Université Bordeaux 1, Avenue du Docteur A. Schweitzer, 33608 Pessac Cedex, France.
}

* To whom correspondence should be addressed. Fax: 33-5-40002761. E-mail: chevalie@icmcb-bordeaux.cnrs.fr 


\section{Abstract}

The hydrogenation process of the antiferromagnetic compounds CePdIn and CePdSn has been studied. Investigation of the new hydrides CePdInH and CePdSnH by X-ray powder diffraction shows that they adopt the same crystal symmetry as the original intermetallic but the unit cell volume increases during the hydrogenation. Magnetization, electrical resistivity, thermoelectric power and ${ }^{119} \mathrm{Sn}$ Mössbauer spectroscopy measurements reveal that CePdInH and CePdSnH order antiferromagnetically below $\mathrm{T}_{\mathrm{N}}=3.0(2) \mathrm{K}$ and $5.0(2) \mathrm{K}$ respectively. Moreover, the physical properties of the hydrides are less influenced by the Kondo effect. The changes of Néel temperature involved by $\mathrm{H}$-insertion are explained on the basis of the Doniach’s diagram considering the competition between Kondo and RKKY interactions.

Key Words : Cerium compounds, Hydrogenation, Magnetism, Kondo effect.

PACS numbers : 75.12.Jf; 75.30.Mb; 75.50.Ee

\section{Introduction}

Many intermetallics based on cerium are considered as strongly correlated electrons systems (SCES) [1]. Their interesting physical properties such as valence fluctuation or heavy fermions behaviour, superconductivity, semiconductivity, non-Fermi liquid behaviour and quantum phase transition result from the competition between the RKKY indirect interaction and the Kondo effect. These interactions depend on the coupling constant $\mathrm{J}_{\mathrm{cf}}$ between $4 \mathrm{f}(\mathrm{Ce})$ and conduction electrons and are defined by two temperatures : the indirect magnetic RKKY temperature $\mathrm{T}_{\mathrm{RKKY}}$ proportional to $\mathrm{J}_{\mathrm{cf}}{ }^{2}$ and the Kondo temperature $\mathrm{T}_{\mathrm{K}}$ which exhibits an exponential dependence on $\mathrm{J}_{\mathrm{cf}}$. The competition between $\mathrm{T}_{\mathrm{RKKY}}$ and $\mathrm{T}_{\mathrm{K}}$ temperatures versus $\mathrm{J}_{\mathrm{cf}}$ is qualitatively described by the well-known Doniach's phase diagram presented in Fig. 1 [2]. $\mathrm{J}_{\mathrm{cf}}$ depends both on the $4 \mathrm{f}(\mathrm{Ce})$-conduction electrons mixing (i. e. $\mathrm{V}_{\mathrm{cf}}$ ) and on the location of the $4 \mathrm{f}(\mathrm{Ce})$ levels (i. e. $\mathrm{E}_{4 \mathrm{f}}$ ) relative to Fermi level (i. e. $\mathrm{E}_{\mathrm{F}}$ ) and so $\mathrm{J}_{\mathrm{cf}}$ is proportional to $\mathrm{V}_{\mathrm{cf}}{ }^{2} /\left(\mathrm{E}_{\mathrm{F}}-\mathrm{E}_{4 \mathrm{f}}\right)$. Hence three ranges can be distinguished on the Doniach's diagram : (i) if the $4 \mathrm{f}(\mathrm{Ce})$ levels lie deep below $\mathrm{E}_{\mathrm{F}}, \mathrm{J}_{\mathrm{cf}}$ is small and the $4 \mathrm{f}(\mathrm{Ce})$ electrons stay in a normal localized state with magnetic moment (magnetic RKKY metal); (ii) when $\mathrm{E}_{4 \mathrm{f}}$ approaches $\mathrm{E}_{\mathrm{F}}, \mathrm{J}_{\mathrm{cf}}$ increases and the compound begins to exhibit Kondo-like behaviour inducing a reduction of the ordered temperature and the magnetic moment (magnetic Kondo system); (iii) with further 
increasing $\mathrm{J}_{\mathrm{cf}},\left(\mathrm{J}_{\mathrm{cf}}>\left(\mathrm{J}_{\mathrm{cf}}\right)_{\mathrm{c}}\right)$, the system goes into the non-magnetic valence fluctuation regime (non-magnetic Kondo system).

Using their low-temperature properties, Fujita et al. have located CeTIn and CeTSn (T $=\mathrm{Ni}$ or Pd) compounds on the Doniach's diagram [3]. The two ternary compounds based on nickel does not exhibit magnetic ordering: (i) CeNiIn is a valence fluctuating system having a high Kondo temperature (i. e. $\mathrm{T}_{\mathrm{K}}=94 \mathrm{~K}$ ) estimated from specific heat measurements [4]; (ii) CeNiSn is an anisotropic dense Kondo compound forming a pseudogap in the density of states at the Fermi level [5]. Moreover, CeNiSn is close to the critical value $\left(J_{\mathrm{cf}}\right)_{\mathrm{c}}$ as the application of uniaxial pressure [6] or small substitution of $\mathrm{Pd}$ for $\mathrm{Ni}$ [7] induces the occurrence of an antiferromagnetic ordering. On the contrary, the palladium based ternary compounds show antiferromagnetic ordering : (i) CePdIn is a Kondo antiferromagnet with its Kondo temperature $T_{K}=3.3 \mathrm{~K}$ is higher than its Néel temperature $T_{N}=1.7 \mathrm{~K}[4,8]$; (ii) CePdSn presents both a high ordered temperature $\mathrm{T}_{\mathrm{N}}=7.5 \mathrm{~K}$ and a smaller influence of the Kondo effect $[9,10]$. (This last point justifies that CePdSn is presented in Fig. 1 closed to the limit between the magnetic RKKY metal and the magnetic Kondo system). The replacement of Ni by bigger Pd in both CeNiIn and CeNiSn compounds induces the occurrence of a magnetic ordering. This agrees with the increase of the unit cell volume leading to a decrease of $\mathrm{J}_{\mathrm{cf}}$. Therefore, this last decrease is expected owing to “chemical expansion” effect.

Recently, we have shown that the hydrogenation of the intermetallics CeNiX (X $=\mathrm{Al}$, $\mathrm{Ga}, \mathrm{In}, \mathrm{Ge}$ and $\mathrm{Sn}$ ) also induces a decrease of $\mathrm{J}_{\mathrm{cf}}$ [11]. For instance, the insertion of hydrogen into the sample of CeNiIn and CeNiSn reveals : (i) an increase of the unit cell volume, respectively $9.6 \%$ and $7.85 \%$; (ii) a ferromagnetic transition below $\mathrm{T}_{\mathrm{C}}=6.8(2) \mathrm{K}$ $\left(\mathrm{CeNiInH}_{1.8(1)}\right)$ and $\mathrm{T}_{\mathrm{C}}=7.0(2) \mathrm{K}\left(\mathrm{CeNiSnH}_{1.8(2)}\right)[12,13]$. In other words, the hydrogenation which induces the transition from an intermediate valence state (CeNiIn) or Kondo insulator behaviour (CeNiSn) to a ferromagnetic ordering, can be considered as an application of “negative” pressure on intermetallics.

In this view, it is interesting to investigate the influence of the hydrogenation on the antiferromagnetic ordering of CePdIn and CePdSn. Considering the role of the Kondo effect on the physical properties of these two compounds, it could be assumed that the H-insertion into CePdIn and CePdSn will induce respectively an increase and a decrease of the Néel temperature. In this paper, the crystallographic, electrical, thermoelectric and magnetic properties of the new hydrides $\mathrm{CePdInH}_{1.0(1)}$ and $\mathrm{CePdSnH}_{1.0(1)}$ are reported. Also, the 
investigation of $\mathrm{CePdSnH}_{1.0(1)}$ by ${ }^{119} \mathrm{Sn}$ Mössbauer spectroscopy is presented and compared to that of CePdSn [14].

\section{Experimental procedures}

The samples CePdIn and CePdSn were synthesized by arc-melting of a stoichiometric mixture of pure elements (purity above 99.9\%) in a high purity argon atmosphere. Then, the samples were turned and remelted several times to ensure homogeneity. An annealing treatment was done for one month at $1073 \mathrm{~K}$ by enclosing the sample in evacuated quartz tubes.

Hydrogen absorption experiments were performed using the apparatus described previously [15]. An ingot of an annealed sample was heated under vacuum at $523 \mathrm{~K}$ for 12 hours and then exposed for $48 \mathrm{~h}$ to $4 \mathrm{MPa}$ of hydrogen gas. The amount of absorbed hydrogen was determined volumetrically by monitoring pressure changes in a calibrated volume.

X-ray powder diffraction with the use of a Philips 1050-diffractometer $(\mathrm{Cu} \mathrm{K \alpha}$ radiation) was applied, before and after hydrogenation, for the characterization of the structural type and for the phase identification of the samples. The unit cell parameters were determined by a least-squares refinement method using silicon (5N) as an internal standard. The crystal structures of the samples before and after hydrogenation were determined by the Rietveld profile refinement using the Fullprof software [16].

Magnetization measurements were performed using a Superconducting QUantum Interference Device (SQUID) magnetometer in the temperature range 1.8-370 K and applied fields up to $5 \mathrm{~T}$. Electrical resistivity was determined above $4.2 \mathrm{~K}$ on a polycrystalline sample using standard dc four-probe measurements. For this investigation, the hydrides were compacted at room temperature (compactness $\cong 80 \%$ ) in order to form a pellet (diameter $=6$ $\mathrm{mm}$ and thickness $=3 \mathrm{~mm}$ ) and then heated for $48 \mathrm{~h}$ at $523 \mathrm{~K}$ under a $4 \mathrm{MPa}$ pressure of hydrogen. Thermoelectric power measurements were performed on the same pellet using a dynamic method. Details of the cell used and measurement methods have been described previously [17].

${ }^{119} \mathrm{Sn}$ Mössbauer measurements were performed on CePdSn and its hydride between 4.2 and $300 \mathrm{~K}$ using a $\mathrm{CaSnO}_{3}$ source and a conventional constant acceleration spectrometer. The isomer shifts (IS) were quoted relative to that of $\mathrm{CaSnO}_{3}$ measured at room temperature. The samples contain $15 \mathrm{mg}$ natural tin per $\mathrm{cm}^{2}$. The spectra were fitted to the sum of 
Lorentzians by least square refinements. All calculations were carried out without any constraint on the amplitudes and the widths.

\section{Results and discussion}

3.1. Preparation of hydrides and structural properties

Under the experimental conditions described previously $\left(\mathrm{T}=523 \mathrm{~K}\right.$ and $\mathrm{P}\left(\mathrm{H}_{2}\right)=4$ MPa), CePdIn and CePdSn absorb hydrogen; the hydrogenation induces a pulverisation of the starting ingots. The amount of H-atom inserted is 1.0(1) per CePdIn or CePdSn formula. These hydrides are stable in ambient conditions.

The investigation of $\mathrm{CePdInH}_{1.0}$ and $\mathrm{CePdSnH}_{1.0}$ by $\mathrm{X}$-ray powder diffraction reveals that the hydrides exhibit the same crystal symmetry as the original intermetallic. The unit cell parameters of these two series are compared in table 1. It is important to note that the hydrogenation increases significantly the unit cell volume V; respectively 3.5 and $2.5 \%$ after H-insertion into the CePdIn and CePdSn lattices.

$\mathrm{CePdInH}$ crystallises as the other hydrides $\mathrm{RENiInH}_{\mathrm{X}}(\mathrm{RE}=\mathrm{La}, \mathrm{Ce}, \mathrm{Nd})$ in the hexagonal ZrNiAl-type structure where $\mathrm{H}$-atoms occupy the $\left[\mathrm{RE}_{3} \mathrm{Ni}\right]$ tetrahedral sites $[12,18$, 19]. However, the hydrogenation of CePdIn causes a pronounced anisotropic expansion of the unit cell: the a-parameter increases weakly $(0.1 \%)$ whereas the c-parameter increases strongly (3.2 \%). For instance, the comparison of the X-ray powder pattern of CePdIn and its hydride (Fig. 2), evidences after hydrogenation a very small decrease of $2 \theta$ for Bragg reflections of a-depending planes (300) and a strong decrease for c-depending planes (002). Similar behaviour was observed during the hydrogenation of CeNiIn and was connected with the location of the $\mathrm{H}$-atoms in the $\left[\mathrm{Ce}_{3} \mathrm{Ni}\right]$ tetrahedral sites along the c-axis. The full occupancy of these tetrahedral sites leads to the formation of the hydride $\mathrm{RENiInH}_{1.33}[18$, 19]. In $\mathrm{CePdInH}$, the obtained formula indicates that only $75 \%$ of the sites [ $\left.\mathrm{Ce}_{3} \mathrm{Pd}\right]$ are occupied. Similar result was observed for the deuterides $\operatorname{RENiInD}_{\mathrm{x}}(\mathrm{RE}=\mathrm{La}, \mathrm{Ce}, \mathrm{Nd})$ demonstrating that the [RE $\left.{ }_{3} \mathrm{Ni}\right]$ sites can be partially filled [18].

CePdSnH is isomorphous to the hydride CeNiSnH showing a significant deformation of the orthorhombic TiNiSi-type structure [12, 20, 21]. In these hydrides, the cerium threedimensional network can be described by an intergrowth of distorted trigonal $\left[\mathrm{Ce}_{6}\right]$ prisms surrounding alternatively the $\mathrm{Ni}$ (or Pd) and Sn atoms. Moreover, in this structure, two types of chemically similar but geometrically different $\left[\mathrm{Ce}_{3} \mathrm{Ni}\right.$ or $\left.\mathrm{Ce}_{3} \mathrm{Pd}\right]$ tetrahedra are formed; only one that has a nearly regular $\mathrm{Ce}_{3}$ side is fully occupied by a hydrogen atom [20]. The 
other significantly deformed $\left[\mathrm{Ce}_{3} \mathrm{Ni}\right.$ or $\left.\mathrm{Ce}_{3} \mathrm{Pd}\right]$ tetrahedral is empty. This hydrogen occupation, agrees with the anisotropic expansion of the unit cell (Table 1 : a- and bparameters decrease respectively of -1.6 and $-3.6 \%$ whereas c-parameter increases strongly of $8.0 \%$ ) as explained in the case of CeNiSnH [20].

In order to obtain more information on the evolution of the structural properties after hydrogenation, the $\mathrm{CePdSnH}_{\mathrm{y}}$ system ( $\mathrm{y}=0$ and 1) has been investigated by ${ }^{119} \mathrm{Sn}$ Mössbauer spectroscopy. The spectra recorded at $293 \mathrm{~K}$ are shown in Fig. 3. The spectrum for CePdSn can be fitted as a symmetric quadrupole split doublet. QS $=0.71(1) \mathrm{mm} / \mathrm{s}$ with a line-width at half-height of $\Gamma=0.97(1) \mathrm{mm} / \mathrm{s}$. The isomer shift was determined equal to IS $=1.89(1) \mathrm{mm} / \mathrm{s}$. The quadrupole splitting originates from the non-cubic local symmetry of Sn sites. The value of QS determined here is similar to that previously reported for this ternary stannide (QS = $0.73 \mathrm{~mm} / \mathrm{s}$ ) [14]. The value of IS is typical for a metal type Sn. For example in the parent stannide CeNiSn crystallizing also in the orthorhombic TiNiSi-type, IS $=1.900(6) \mathrm{mm} / \mathrm{s}$ at $293 \mathrm{~K}$ [22]. A symmetric quadrupole split doublet is also observed for the hydride CePdSnH; the Mössbauer parameters being IS $=1.94(1) \mathrm{mm} / \mathrm{s}$, QS $=0.95(1) \mathrm{mm} / \mathrm{s}$ and $\Gamma=0.98(1) \mathrm{mm} / \mathrm{s}$. At $293 \mathrm{~K}$, IS increases in the sequence CePdSn $\rightarrow \mathrm{CePdSnH}$ indicating an increase of the selectron density at the Sn nuclei. Similarly, QS increases in agreement with the presence of $\mathrm{H}$-atoms surrounding the Sn nuclei. In the other words, insertion of hydrogen in the CePdSn lattice modifies strongly the ${ }^{119} \mathrm{Sn}$ Mössbauer signals. Similar behavior was reported by us during the study of the sequence CeNiSn $\rightarrow$ CeNiSnH [22].

\subsection{Magnetic and electrical properties of CePdInH}

Fig. 4 compares the thermal dependence of the reciprocal magnetic susceptibility $\chi_{\mathrm{m}}{ }^{-1}$ of CePdIn and its hydride. Above $150 \mathrm{~K}$, the data for CePdIn can be fitted with a Curie-Weiss law $\chi_{\mathrm{m}}{ }^{-1}=\left(\mathrm{T}-\theta_{\mathrm{p}}\right) /\left(0.125 \mu_{\mathrm{eff}}^{2}\right)$ giving $\theta_{\mathrm{p}}=-42(1) \mathrm{K}$ as paramagnetic Curie temperature and $\mu_{\text {eff }}=2.59(2) \mu_{\mathrm{B}} / \mathrm{Ce}$ as magnetic effective moment (Table 1 ). These values are close to that reported previously (Table 1 ) [8]. The $\chi_{\mathrm{m}}{ }^{-1}=\mathrm{f}(\mathrm{T})$ curve concerning the hydride CePdInH follows also above $100 \mathrm{~K}$ a Curie-Weiss law with $\theta_{\mathrm{p}}=-28(1) \mathrm{K}$ and $\mu_{\mathrm{eff}}=2.53(2) \mu_{\mathrm{B}} / \mathrm{Ce}$. This last value agrees with that calculated for a free $\mathrm{Ce}^{3+}$ ion $\left(2.54 \mu_{\mathrm{B}}\right)$. The increase of $\theta_{\mathrm{p}}$ from 42(1) $\mathrm{K}$ to $-28(1) \mathrm{K}$ in the sequence CePdIn $\rightarrow \mathrm{CePdInH}$ suggests that the hydrogenation induces a decrease of the Kondo interaction.

Above $1.8 \mathrm{~K}$, no anomaly attributed to the occurrence of a magnetic ordering transition can be detected by magnetization measurements performed on CePdIn (Fig. 5). This 
result confirms the low Néel temperature $T_{N}=1.65 \mathrm{~K}$ of this ternary indide determined by specific heat measurements $[4,8]$. On the contrary, the magnetic susceptibility of CePdInH exhibits a kink at $\mathrm{T}_{\mathrm{N}}=3.0(2) \mathrm{K}$. This behaviour indicates that the hydride presents an antiferromagnetic ordering with a Néel temperature almost twice greater than that determined for the initial intermetallic. Similar increase of the magnetic ordering temperature after hydrogenation was evidenced for CePtAl; the Curie temperature increases from $5.6 \mathrm{~K}$ to 11.6 K after H-insertion [23].

As shown in Fig. 6, the reduced electrical resistivity of CePdIn, measured between 4.2 $\mathrm{K}$ and $270 \mathrm{~K}$, is strongly modified by its hydrogenation. The curve $\rho(\mathrm{T}) / \rho(270 \mathrm{~K})=\mathrm{f}(\mathrm{T})$ for CePdIn reveals several anomalies : (i) a broad maximum occurs around $64 \mathrm{~K}$; (ii) below this maximum, $\rho(T)$ decreases and a minimum appears near $18 \mathrm{~K}$; (iii) finally, below this minimum there is a rise of $\rho(\mathrm{T})$ down to $4.2 \mathrm{~K}$ (inset in Fig. 6). In the two temperature ranges 80-190 $\mathrm{K}$ and 4.2-12 K, the law $\rho(\mathrm{T}) / \rho(270 \mathrm{~K})=-\mathrm{A} \log \mathrm{T}(\mathrm{A}=$ constant $)$ is observed. Such behaviors which agree with those reported for a polycrystalline sample of CePdIn [8, 24], is expected for Kondo-type interactions in the presence of crystal field effects [25]. The hightemperature logarithmic regime represents the Kondo effect in the excited doublet, whereas the low-temperature region is relative to the Kondo effect from the crystal field ground state (incoherent Kondo scattering). As the measurements were performed above $4.2 \mathrm{~K}$ (i.e. higher than the Néel temperature $T_{N}=1.65 \mathrm{~K}$ ), no decrease is evidenced at lower temperature. The curve for the hydride CePdInH shows different characteristics : (i) a downward curvature around 60-80 K associated with a crystal field effect; (ii) a minimum at $14 \mathrm{~K}$ and a maximum near $6 \mathrm{~K}$. The minimum at $18 \mathrm{~K}$ or $14 \mathrm{~K}$ for CePdIn and its hydride respectively is relative to the Kondo effect from the crystal field ground state. Such decrease (18 K $\rightarrow 14 \mathrm{~K}$ ) indicates that the Kondo effect decreases with H-insertion. Also, the decrease of $\rho(\mathrm{T})$ below $6 \mathrm{~K}$ for CePdInH could be associated to the loss of spin disorder scattering of the conduction electrons owing to the appearance of an antiferromagnetic transition (detected around 3.0(2) K as shown previously by magnetization measurement).

Fig. 7 shows the temperature dependence of thermoelectric power S of CePdInH. This curve $S=f(T)$ exhibits many similarities to that reported for the initial indide CePdIn [26]. It is mainly characterized by the existence of two extremes : a positive maximun of about 9 $\mu \mathrm{V} / \mathrm{K}$ near $90 \mathrm{~K}$ and a negative one at $-2.5 \mu \mathrm{V} / \mathrm{K}$ around $16 \mathrm{~K}$. Between that, the thermoelectric power is equal to 0 at $28 \mathrm{~K}$. Such behavior is commonly observed for Ce-based Kondo-lattice systems having a low Kondo temperature as $\mathrm{CeAl}_{2}$ [27]. The broad positive 
peak is ascribed to an interplay of the Kondo and the crystalline electric field (CEF) effects [28]. The peak height measures the strength of Kondo effect, and the peak temperature measures the CEF splitting. The temperature $\cong 90 \mathrm{~K}$ of the positive maximum for CePdInH, is comparable to that where the curve $\rho(T) / \rho(270 \mathrm{~K})=\mathrm{f}(\mathrm{T})$ (Fig. 6) exhibits a downward curvature maximum. Meanwhile, the origin of the negative peak $(16 \mathrm{~K})$ is ascribed by Sakurai et al. [29] to an interplay of the Kondo effect and the onset of the short-range magnetic correlation. Finally, it is interesting to note that the value of $S \cong 32 \mu \mathrm{V} / \mathrm{K}$ for the broad positive peak in CePdIn [26] is higher than that determined here $(9 \mu \mathrm{V} / \mathrm{K})$ for $\mathrm{CePdInH}$, suggesting a diminution of the strength of Kondo effect.

The magnetization and transport measurements performed on the hydride CePdInH reveal that the $\mathrm{H}$-insertion in CePdIn decreases the influence of the Kondo effect and consequently the Néel temperature increases.

\subsection{Magnetic and electrical properties of $\mathrm{CePdSnH}$}

The curves $\chi_{\mathrm{m}}{ }^{-1}=\mathrm{f}(\mathrm{T})$ relative to the reciprocal magnetic susceptibility of CePdSn and CePdSnH are shown in Fig. 8. Above $100 \mathrm{~K}$, the data are on straight lines, obeying the CurieWeiss law. The effective moment $\mu_{\text {eff }}$ for CePdSn and CePdSnH are 2.56(2) $\mu_{\mathrm{B}} / \mathrm{Ce}$ and 2.74(2) $\mu_{\mathrm{B}} / \mathrm{Ce}$ respectively (Table 1 ) which is close to the value of free $\mathrm{Ce}^{3+}$. The deviation from the Curie-Weiss law below $100 \mathrm{~K}$ is due to the crystal electric field effect. In this case also, $\theta_{\mathrm{p}}$ shows a significant decrease in the magnitude considering the sequence CePdSn $\rightarrow$ CePdSnH (Table 1). This may be due to a decrease of the hybridisation effect between $4 \mathrm{f}(\mathrm{Ce})$-electrons and conduction ones.

The magnetic susceptibility $\chi_{\mathrm{m}}$ at low temperatures for CePdSnH and CePdSn is plotted in Fig. 9. The peaks of $\chi_{m}=f(T)$ are identified as the Néel temperature $T_{N}$. The hydrogenation of CePdSn induces a decrease of $\mathrm{T}_{\mathrm{N}}$ from 7.2(2) K to 5.0(2) K. Certainly, the hydrogenation changes also the magnetic structure of CePdSn. At $2 \mathrm{~K}$ and up to $\mu_{0} \mathrm{H} \leq 4.8 \mathrm{~T}$, in the antiferromagnetic range, the magnetization of CePdSn increases linearly with the applied magnetic field (Fig. 10). On the contrary, below $\mathrm{T}_{\mathrm{N}}=5.0(2) \mathrm{K}$ the magnetization of the hydride CePdSnH increases linearly at low fields, shows a steep rise in the range 0.6-1.4 T and then exhibits a tendency to saturation. This behaviour is typical of a magnetic transition induced by the applied magnetic field; at $2 \mathrm{~K}$ and $4 \mathrm{~K}$, the critical field is estimated respectively at $1.2(2)$ and $0.8(2) \mathrm{T}$. 
Above $30 \mathrm{~K}$, the reduced resistivity of CePdSn (Fig. 11) does not vary linearly with temperature (as for a normal metal) and the curve $\rho(T) / \rho(270 \mathrm{~K})=\mathrm{f}(\mathrm{T})$ presents a downward curvature associated with a crystal-field effect which becomes temperature dependent in the paramagnetic range. At low temperatures its reduced resistivity increases slightly between 20 $\mathrm{K}$ and 7.3(2) $\mathrm{K}$ then decreases strongly. This behaviour is typical of a magnetically ordered Kondo system showing the presence of crystal field spitting of the $4 \mathrm{f}(\mathrm{Ce})$ electron. No similar anomaly can be detected from the curve $\rho(T) / \rho(270 \mathrm{~K})=\mathrm{f}(\mathrm{T})$ for CePdSnH; a linear variation is observed above $50 \mathrm{~K}$, a tendency to saturation appears between $30 \mathrm{~K}$ and $5 \mathrm{~K}$ where a beginning of decrease occurs (appearance of the antiferromagnetic ordering). This strikingly different behaviour of hydride indicates the absence of Kondo scattering as found in CePdSn.

The curve $S=f(T)$ for CePdSnH (Fig. 7) agrees with a small influence of the Kondo effect on its physical properties. This curve can be compared to that reported previously for the ternary stannide CePdSn [14] where a similar positive peak was observed; but the temperature of this maximum decreases from $110 \mathrm{~K}$ to $60 \mathrm{~K}$ and the value of $\mathrm{S}$ at this peak diminishes from $8 \mu \mathrm{V} / \mathrm{K}$ to $2.5 \mu \mathrm{V} / \mathrm{K}$ in the sequence CePdSn $\rightarrow \mathrm{CePdSnH}$. These two decreases are correlated to the diminution of the Kondo effect during the hydrogenation of CePdSn.

Similar comparison applied on the curves $S=f(T)$ observed for the two hydrides CePdInH and CePdSnH (Fig. 7) shows that the influence of the Kondo effect decreases from CePdInH to CePdSnH. Indeed, the temperature of the positive maximum decreases from $90 \mathrm{~K}$ to $60 \mathrm{~K}$ and the $\mathrm{S}$ value at these temperatures from $9 \mu \mathrm{V} / \mathrm{K}$ to $2.5 \mu \mathrm{V} / \mathrm{K}$.

At $4.2 \mathrm{~K}$, in the antiferromagnetic state, the ${ }^{119} \mathrm{Sn}$ Mössbauer spectra corresponding to CePdSn and CePdSnH (Fig. 12) are very broad in comparison with those obtained at room temperature (Fig. 3) or in the paramagnetic range of these compounds. (For instance, the spectra-width at half-height for hydride CePdSnH increases weakly from $10.7 \mathrm{~K}$ to $6.1 \mathrm{~K}$ $(2.19 \rightarrow 2.25 \mathrm{~mm} / \mathrm{s})$ and strongly at lower temperatures; 2.50 and $2.65 \mathrm{~mm} / \mathrm{s}$ respectively at 4.8 and $4.2 \mathrm{~K}$ ). These broadening can be explained in terms of a transferred magnetic field that would appear if the antiferromagnetic arrangement of Ce moments, leading to create a hyperfine field $\mathrm{H}_{\mathrm{hf}}$ at the Sn nuclei (the spectra-width at half-height increases significantly below 7.5(5) K and 5.5(5) K respectively for CePdSn and CePdSnH, in agreement with the occurrence of their antiferromagnetic ordering). This agrees for CePdSn which presents an incommensurate magnetic structure [10]. However, the sextuplet, characteristic of magnetic hyperfine interaction is still unresolved due to relatively low value of $\mathrm{H}_{\mathrm{hf}}$, respectively equal 
to $1.45 \mathrm{~T}$ and $1.15 \mathrm{~T}$ for CePdSn and its hydride. The determination of the magnetic structure of CePdSnH by neutron powder diffraction is necessary in order to explain this hyperfine field splitting.

\section{Conclusion}

H-insertion into the ternary compounds CePdIn and CePdSn : (i) allows to obtain the new hydrides CePdInH and CePdSnH which crystallize with the same crystal symmetry as the parent compounds; (ii) induces an increase of the unit cell volume, 3.5 and $2.5 \%$ in the case of CePdIn and CePdSn respectively; (iii) modifies their magnetic antiferromagnetic properties, $\mathrm{T}_{\mathrm{N}}$ increases in the sequence CePdIn $\rightarrow$ CePdInH but decrease for CePdSn $\rightarrow$ CePdSnH; (iv) finally, diminishes the influence of the Kondo effect on their physical properties. In other words, in these cases the hydrogenation can be compared to the application of a "negative” pressure inducing a decrease of the $J_{\text {cf }}$ interaction.

The change of the physical properties resulting from the hydrogenation of CeTX compounds ( $\mathrm{T}=\mathrm{Ni}, \mathrm{Pd}$ and $\mathrm{X}=\mathrm{In}, \mathrm{Sn}$ ) can be explained on the basis of the Doniach's diagram (Fig. 1). The hydrogen insertion into : (i) a non-magnetic Kondo system (CeNiIn, CeNiSn) leads to a magnetically ordered hydride $\left(\mathrm{CeNiInH}_{1.8}, \mathrm{CeNiSnH}_{1.8}\right)$; (ii) a magnetic Kondo system strongly (CePdIn) or weakly (CePdSn) influenced by the Kondo effect, induces an increase or a decrease of the Néel temperature.

\section{Acknowledgments}

The authors would like to thank R. Decourt for his assistance during the electrical resistivity and thermoelectric power measurements.

\section{References}

[1] A. S. Edestein, J. Magn. Magn. Mater., 256 (2003) 430.

[2] S. Doniach, Physica B, 91 (1977) 231.

[3] T. Fujita, T. Suzuki, S. Nishigori, T. Takabatake, H. Fujii and J. Sakurai, J. Magn. Magn. Mater., 108 (1992) 35.

[4] K. Satoh, T. Fujita, Y. Maeno, Y. Uwatoko and H. Fujii, J. Phys. Soc. Japan, 59 (1990) 692.

[5] T. Takabatake, F. Teshima, H. Fujii, S. Nishigori, T. Suzuki, T. Fujita, Y. Yamaguchi, J. Sakurai, D. Jaccard, Phys. Rev. B, 41 (1990) 9607. 
[6] K. Umeo, T. Igaue, H. Chyono, Y. Echizen, T. Kosaka and Y. Uwatoko, Phys. Rev. B, 60 (1999) R6957.

[7] M. Kasaya, T. Tani, H. Suzuki, K. Ohoyama and M. Kohgi, J. Phys. Soc. Japan, 60 (1991) 2542.

[8] E. Brück, M. Van Sprang, J. C. P. Klaesse and F. R. De Boer, J. Appl. Phys., 63 (1988) 3417.

[9] D. T. Adroja, S. K. Malik, B. D. Padalia and R. Vijayaraghavan, Solid State Comm., 66 (1988) 1201.

[10] M. Kasaya, T. Tani, K. Ohoyama, M. Kohgi and Y. Isikawa, J. Magn. Magn. Mater., 104-107 (1992) 665.

[11] B. Chevalier, M. Pasturel, J.-L. Bobet, R. Decourt, J. Etourneau, O. Isnard, J. Sanchez Marcos, J. Rodriguez Fernandez, J. Alloys Comp., 383 (2004) 4.

[12] B. Chevalier, M. L. Kahn, J.-L. Bobet, M. Pasturel and J. Etourneau, J. Phys.: Condens. Matter, 14 (2002) L365.

[13] B. Chevalier, J.-L. Bobet, M. Pasturel, E. Bauer, F. Weill, R. Decourt and J. Etourneau, Chem. Mater., 15 (2003) 2181.

[14] J. Sakurai, Y. Yamaguchi, K. Mibu and T. Shinjo, J. Magn. Magn. Mater., 84 (1990) 157.

[15] J.-L. Bobet, S. Pechev, B. Chevalier, B. Darriet, J. Alloys Comp., 267 (1998) 136.

[16] J. Rodriguez-Carvajal, Physica B, 192 (1993) 55.

[17] P. Dordor, E. Marquestaut and G. Villeneuve, Rev. Phys. Appl., 15 (1980) 1607.

[18] V. A. Yartys, R. V. Denys, B. C. Hauback, H. Fjellvag, I. I. Bulyk, A. B. Riabov, YA. M. Kalychak, J. Alloys Comp., 330-332 (2002) 132.

[19] R. V. Denys, A. B. Riabov, V. A. Yartys, B. C. Hauback, H. W. Brinks, J. Alloys Comp., 356-357 (2003) 65.

[20] V. A. Yartys, B. Ouladdiaf, O. Isnard, O. Yu. Khyzhun, K. H. J. Buschow, J. Alloys Comp., 359 (2003) 62.

[21] B. Chevalier, M. Pasturel, J.-L. Bobet, J. Etourneau, O. Isnard, J. Sanchez Marcos and J. Rodriguez Fernandez, J. Magn. Magn. Mat., 272-276 Part 1 (2004) 576.

[22] B. Chevalier, A. Wattiaux, L. Fournès, M. Pasturel, Solid State Sciences, 6 (2004) 573.

[23] J. -L. Bobet, B. Chevalier, F. Weill, J. Etourneau, J. Alloys Comp., 330-332 (2002) 373.

[24] E. Brück, H. Nakotte, K. Bakker, F. R. de Boer, P. F. de Chatel, J.-Y. Li, J. P. Kuang and F.-M. Yang, J. Alloys Comp., 200 (1993) 79. 
[25] B. Cornut and B. Coqblin, Phys. Rev. B, 5 (1972) 4541.

[26] Y. Yamaguchi, J. Sakurai, F. Teshima, H. Kawanaka, T. Takabatake and H. Fujii, J. Phys.: Condens. Matter, 2 (1990) 5715.

[27] C. S. Garde and J. Ray, Phys. Rev. B, 51 (1995) 2960.

[28] A. K. Bhattacharjee and B. Coqblin, Phys. Rev. B, 13 (1976) 3441.

[29] J. Sakurai, H. Takagi, T. Kuwai, Y. Isikawa, J. Magn. Magn. Mater., 177-181 (1998) 407. 


\begin{tabular}{|c|c|c|c|c|c|c|c|c|c|}
\hline \multirow{2}{*}{ Compound } & \multicolumn{5}{|c|}{ Crystallographic data } & \multicolumn{3}{|c|}{ Magnetic data } & \multirow[t]{2}{*}{ Ref. } \\
\hline & Type & a $(\AA)$ & $\mathrm{b}(\AA)$ & c $(\AA)$ & $\mathrm{V}(\AA)^{3}$ & $\begin{array}{c}\mu_{\text {eff }} \\
\left(\mu_{\mathrm{B}} / \mathrm{mol}\right)\end{array}$ & $\theta_{\mathrm{p}}(\mathrm{K})$ & $\mathrm{T}_{\mathrm{N}}(\mathrm{K})$ & \\
\hline CePdIn & $\mathrm{ZrNiAl}$ & 7.698 & & 4.076 & 209.18 & 2.64 & -41 & 1.65 & {$[8]$} \\
\hline CePdIn & ZrNiAl & $7.686(2)$ & & $4.067(1)$ & 208.07 & $2.59(2)$ & $-42(1)$ & & $*$ \\
\hline CePdInH & ZrNiAl & 7.694(2) & & 4.199(1) & 215.27 & $2.53(2)$ & $-28(1)$ & $3.0(2)$ & $*$ \\
\hline CePdSn & TiNiSi & 7.526 & 4.7424 & 7.931 & 283.07 & 2.67 & -68 & 7.5 & [9] \\
\hline CePdSn & TiNiSi & $7.530(2)$ & $4.698(1)$ & $7.957(2)$ & 281.49 & $2.56(2)$ & $-72(1)$ & $7.2(2)$ & $*$ \\
\hline CePdSnH & CeNiSnH & $7.412(2)$ & 4.531(1) & 8.593(2) & 288.59 & $2.74(2)$ & $-29(1)$ & $5.0(2)$ & $*$ \\
\hline
\end{tabular}

* this work

Table 1 Crystallographic and magnetic data relative to the ternary compounds CePdIn and CePdSn and their hydrides. 


\section{Figure captions}

Fig.1 Doniach's diagram applied to the hydrogenation of CeTX compounds with $\mathrm{T}=\mathrm{Ni}$, Pd and $\mathrm{X}=\mathrm{In}$, Sn.

Fig. 2 X-ray powder diffraction patterns of CePdIn and its hydride.

Fig. $3{ }^{119}$ Sn Mössbauer absorption spectra of CePdSn and CePdSnH measured at $293 \mathrm{~K}$. The circles represent experimental values, while the lines are fits of the data.

Fig. 4 Temperature dependence of the reciprocal magnetic susceptibility, measured with an applied field $\mu_{0} \mathrm{H}=4 \mathrm{~T}$, of CePdIn and its hydride.

Fig. 5 Temperature dependence of the magnetic susceptibility, measured with an applied field $\mu_{0} \mathrm{H}=0.1 \mathrm{~T}$, of CePdIn and its hydride.

Fig. 6 Reduced electrical resistivity as a function of log T for CePdIn (inset) and its hydride.

Fig. 7 Temperature dependence of the thermoelectric power of the hydrides CePdInH and CePdSnH.

Fig. 8 Temperature dependence of the reciprocal magnetic susceptibility, measured with an applied field $\mu_{0} \mathrm{H}=4 \mathrm{~T}$, of CePdSn and its hydride.

Fig. 9 Temperature dependence of the magnetic susceptibility, measured with an applied field $\mu_{0} \mathrm{H}=0.1 \mathrm{~T}$, of CePdSnH and CePdSn (inset).

Fig. 10 Field dependence of the magnetization of CePdSn at $2 \mathrm{~K}$ and its hydride at temperatures between $7 \mathrm{~K}$ and $2 \mathrm{~K}$.

Fig. 11 Reduced electrical resistivity as a function of $\log \mathrm{T}$ for CePdSn and its hydride.

Fig. $12{ }^{119}$ Sn Mössbauer absorption spectra of CePdSn and CePdSnH measured at 4.2 K. The circles represent experimental values, while the lines are fits to the data. 


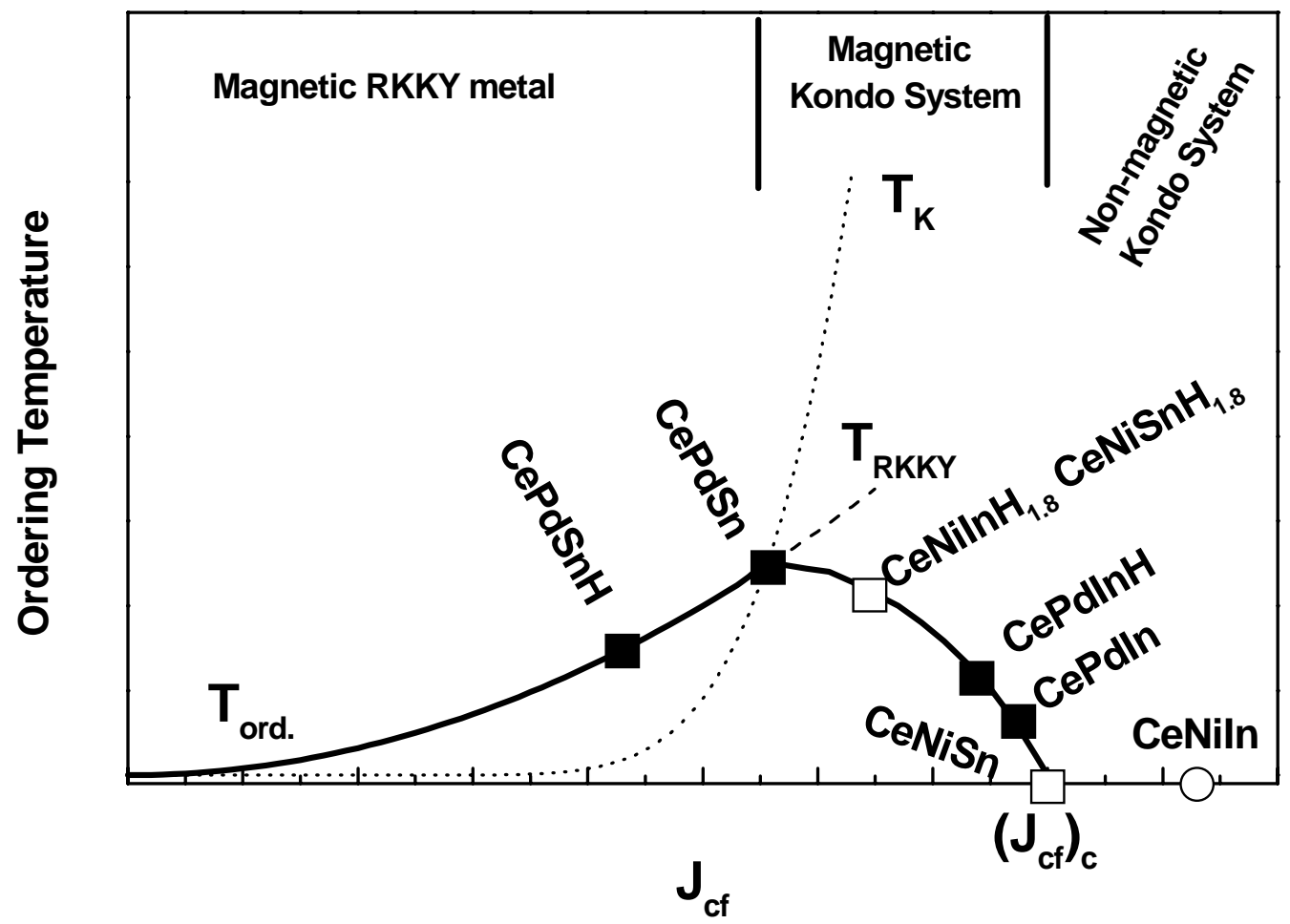

Fig.1 Doniach's diagram applied to the hydrogenation of CeTX compounds with $\mathrm{T}=\mathrm{Ni}$, Pd and $\mathrm{X}=\mathrm{In}$, Sn. 


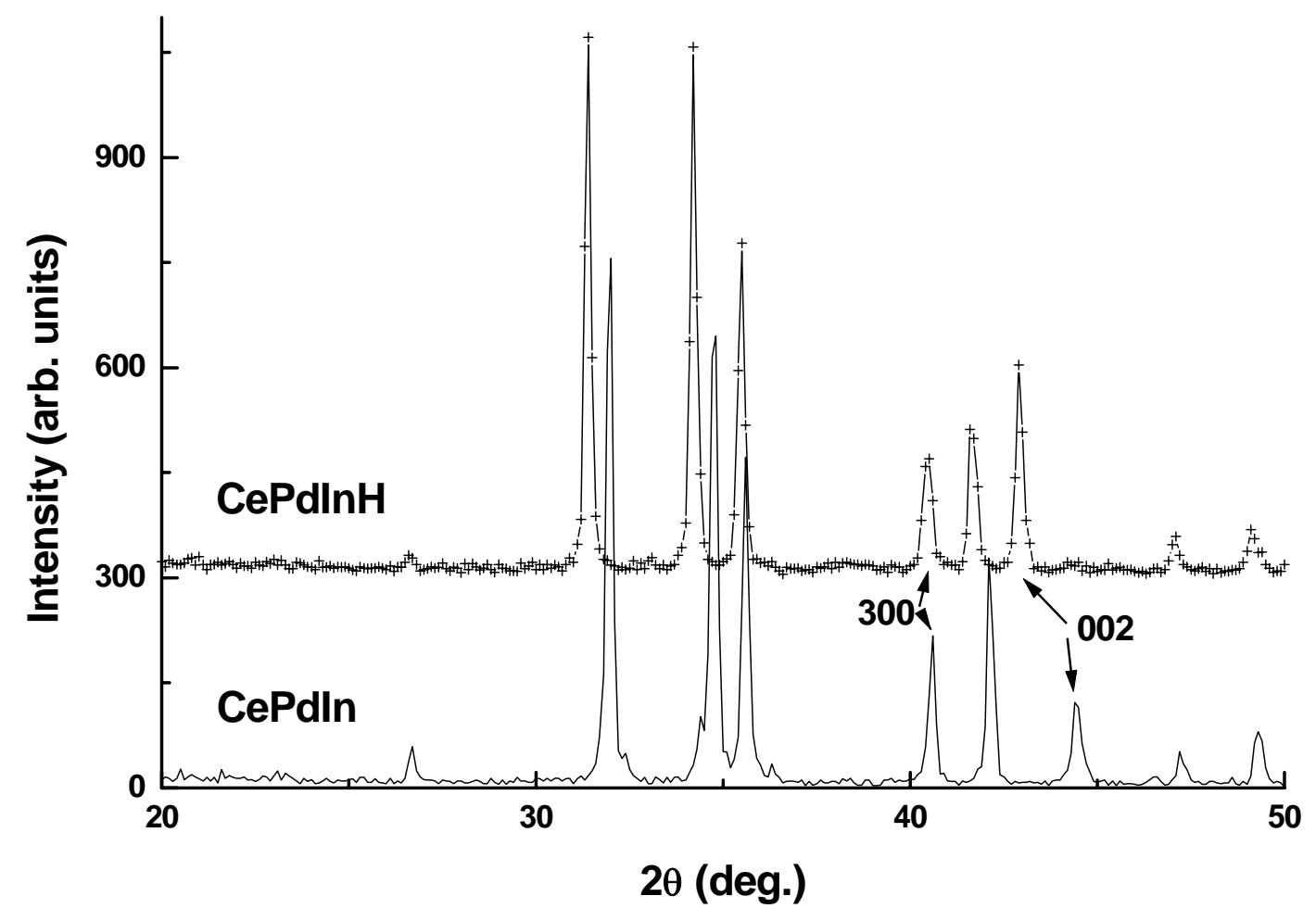

Fig. 2 X-ray powder diffraction patterns of CePdIn and its hydride. 


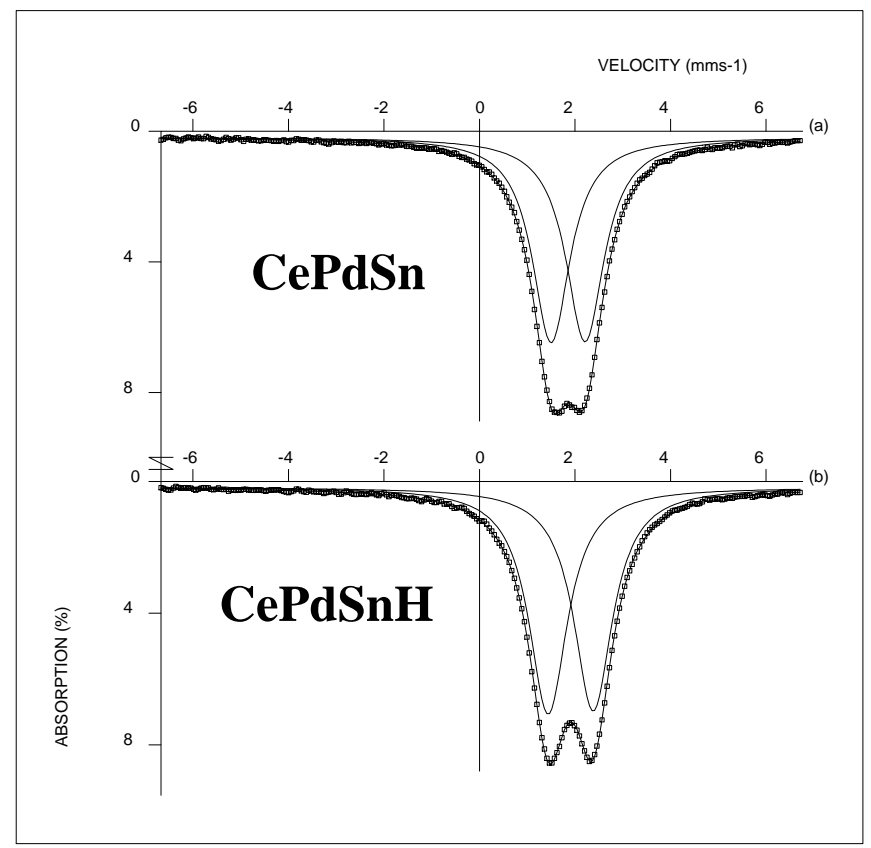

Fig. $3{ }^{119}$ Sn Mössbauer absorption spectra of CePdSn and CePdSnH measured at $293 \mathrm{~K}$. The circles represent experimental values, while the lines are fits of the data. 


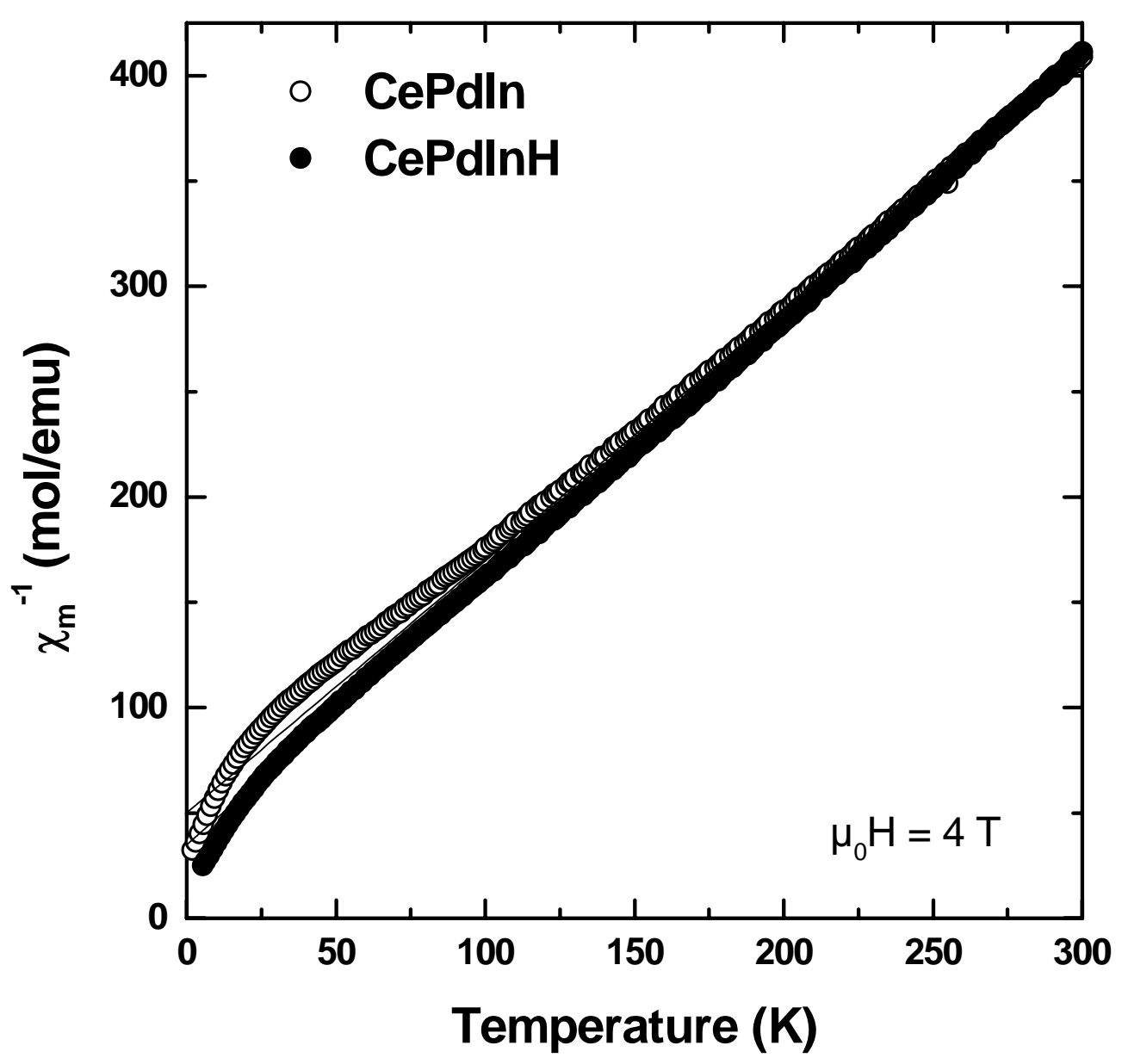

Fig. 4 Temperature dependence of the reciprocal magnetic susceptibility, measured with an applied field $\mu_{0} \mathrm{H}=4 \mathrm{~T}$, of CePdIn and its hydride. 


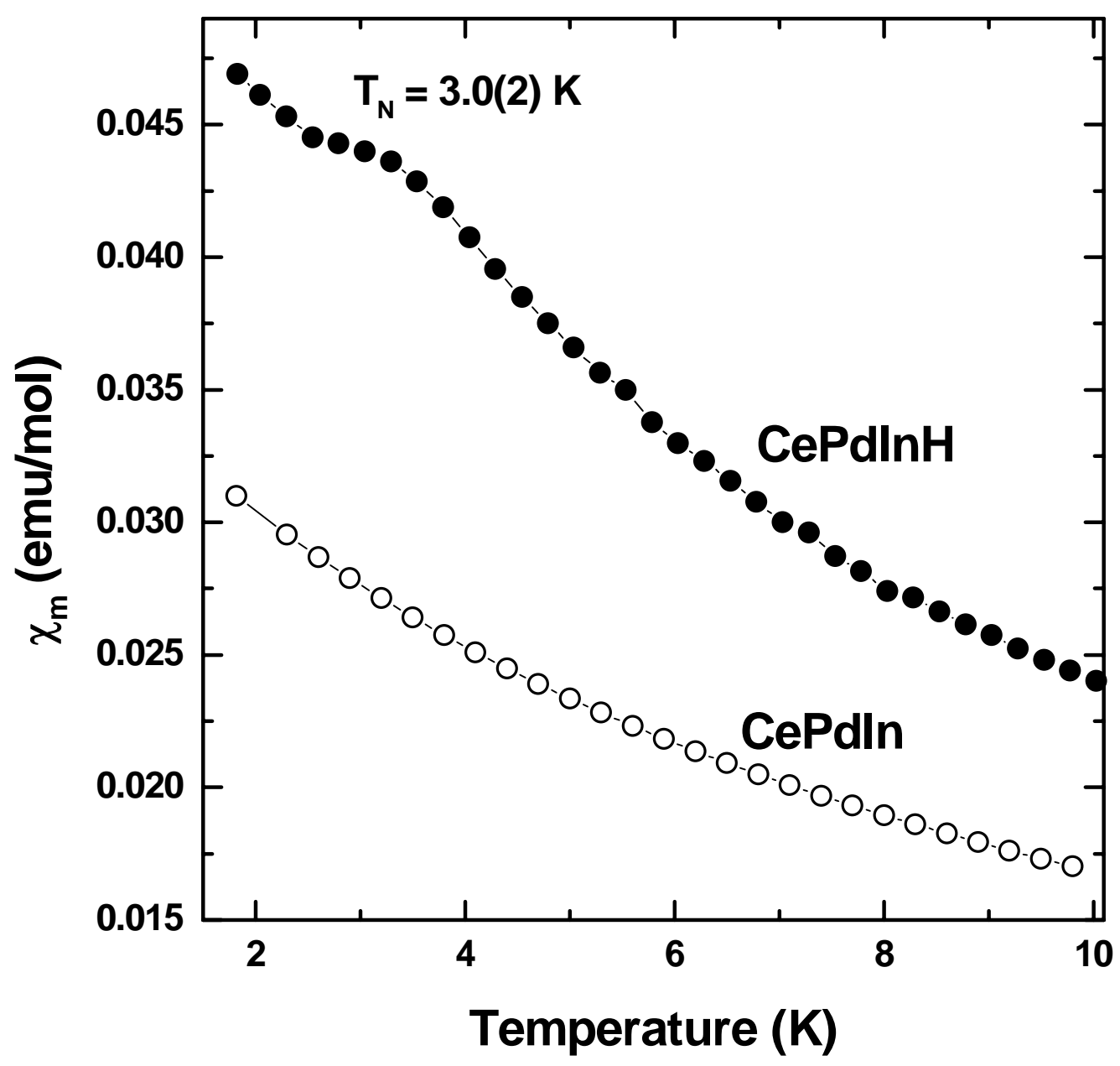

Fig. 5 Temperature dependence of the magnetic susceptibility, measured with an applied field $\mu_{0} \mathrm{H}=0.1 \mathrm{~T}$, of CePdIn and its hydride. 


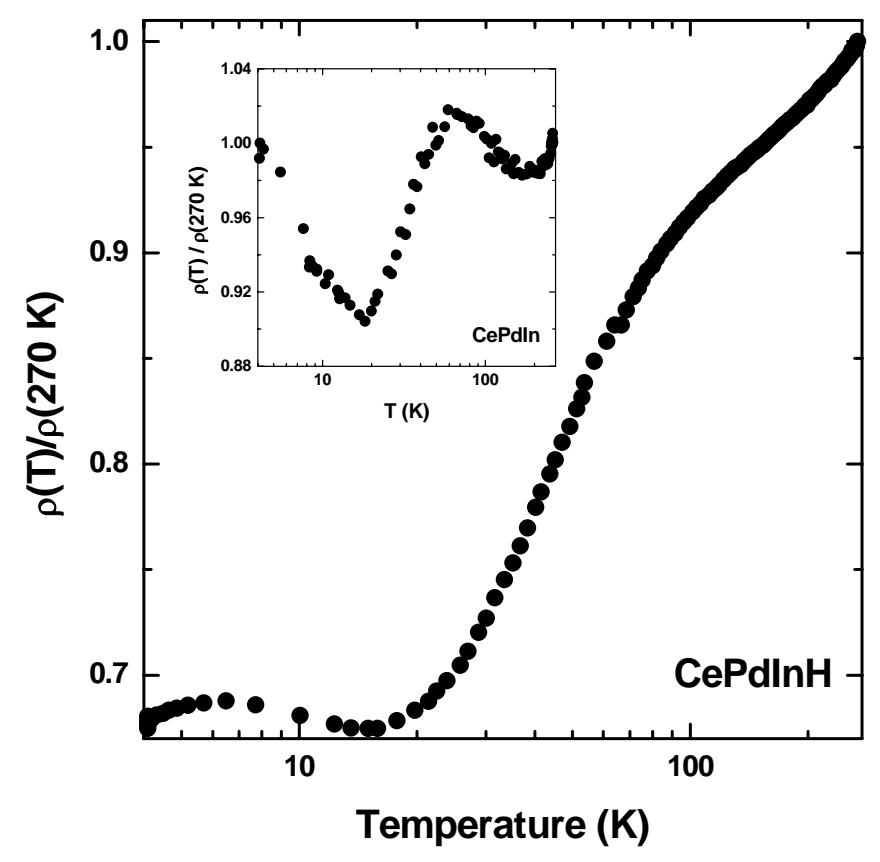

Fig. 6 Reduced electrical resistivity as a function of log T for CePdIn (inset) and its hydride. 


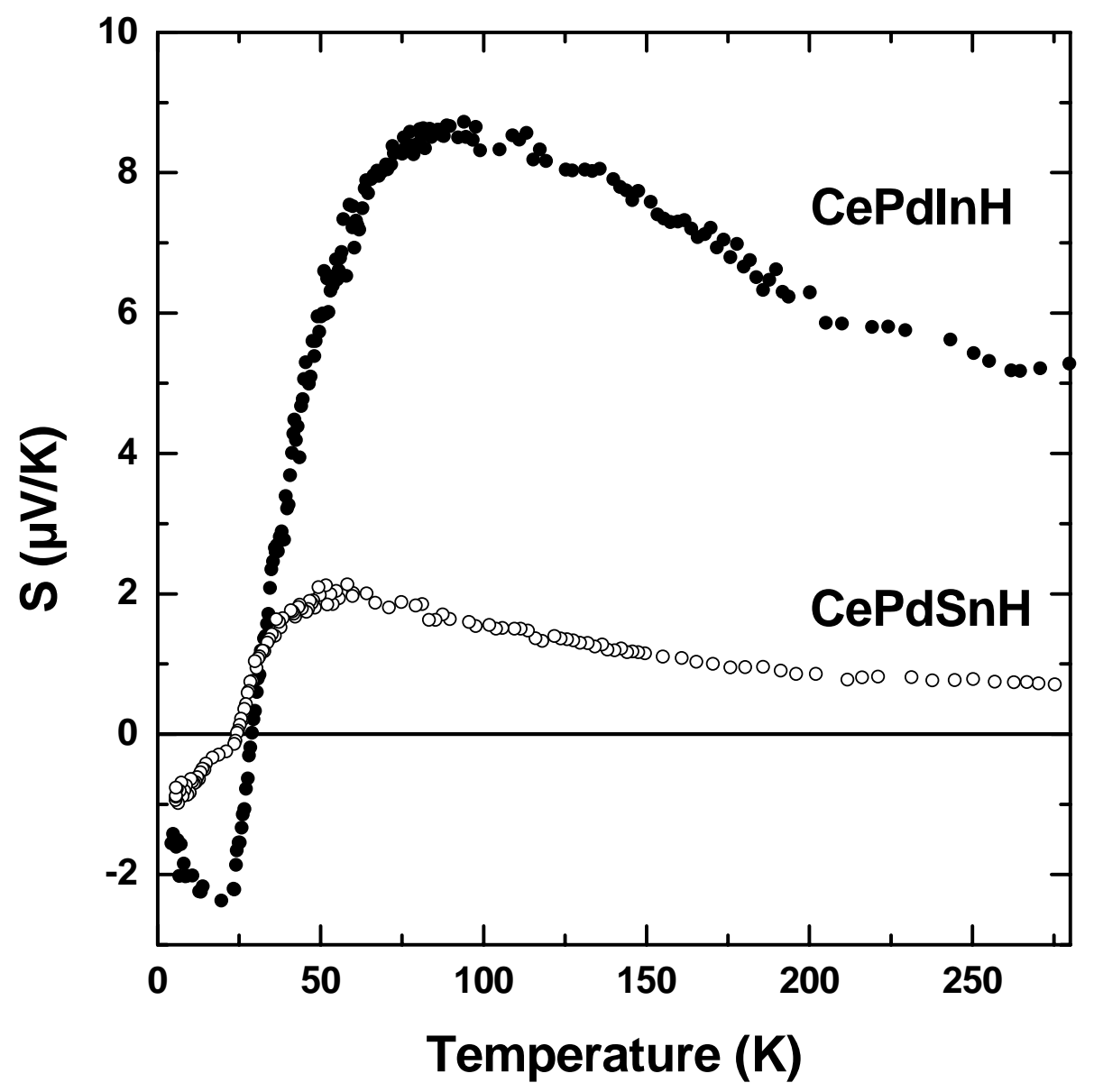

Fig. 7 Temperature dependence of the thermoelectric power of the hydrides CePdInH and CePdSnH. 


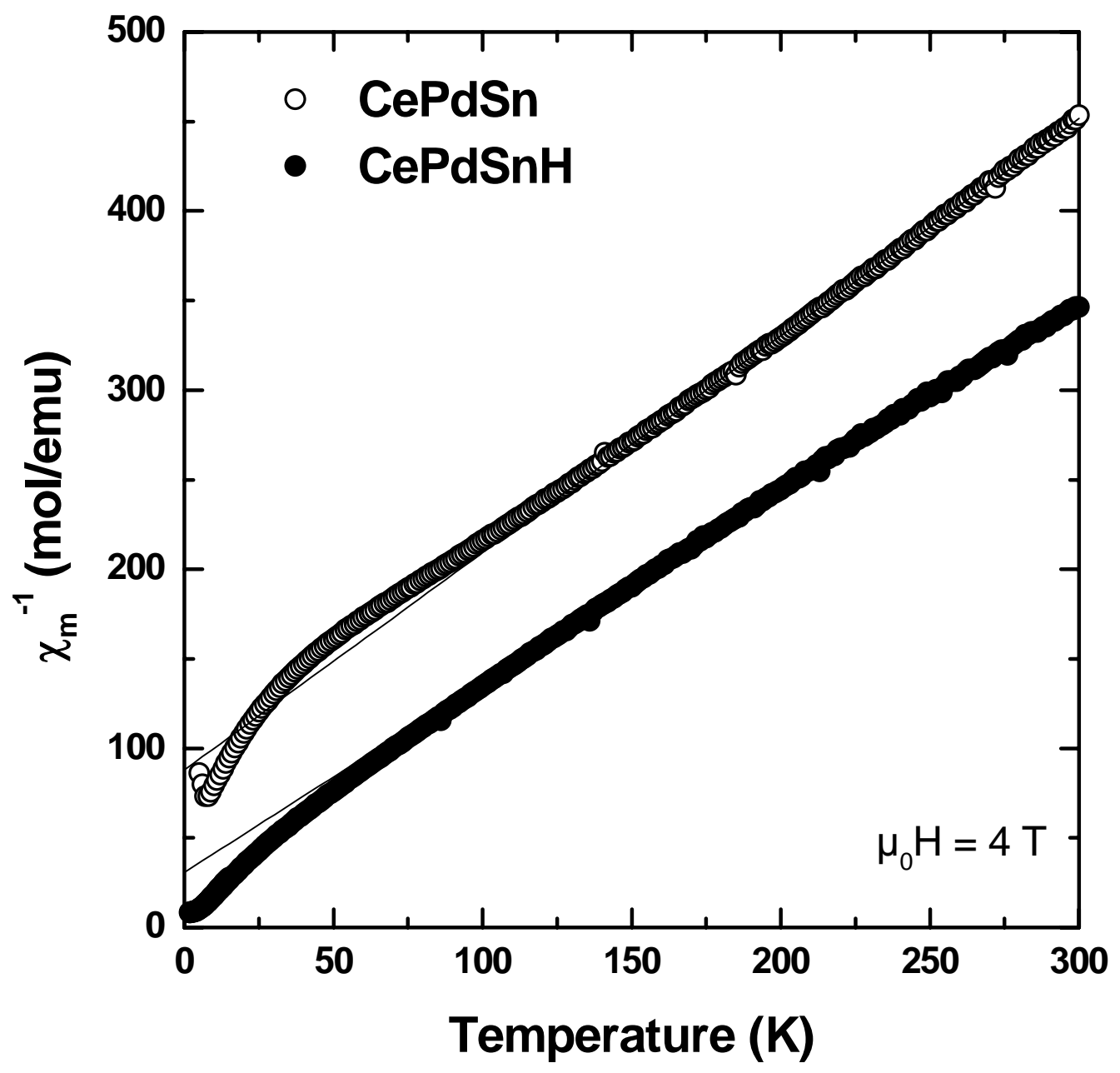

Fig. 8 Temperature dependence of the reciprocal magnetic susceptibility, measured with an applied field $\mu_{0} \mathrm{H}=4 \mathrm{~T}$, of CePdSn and its hydride. 


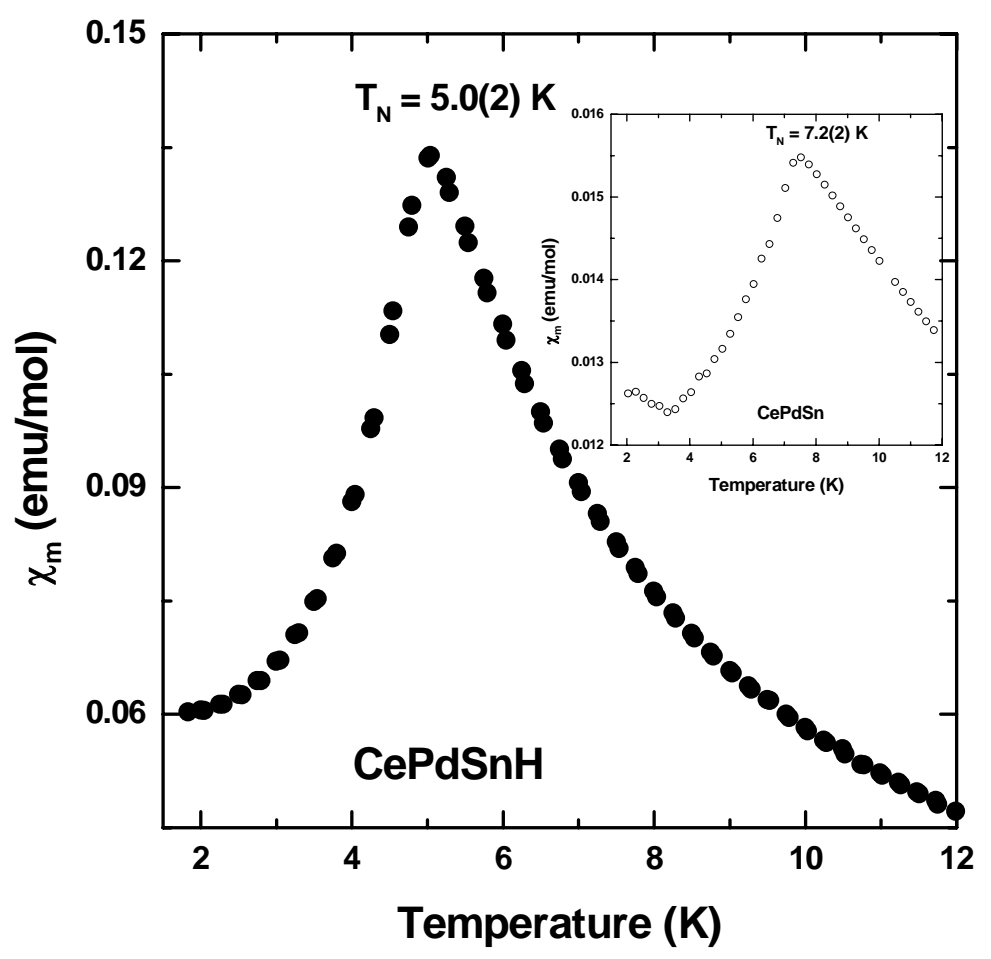

Fig. 9 Temperature dependence of the magnetic susceptibility, measured with an applied field $\mu_{0} \mathrm{H}=0.1 \mathrm{~T}$, of CePdSnH and CePdSn (inset). 


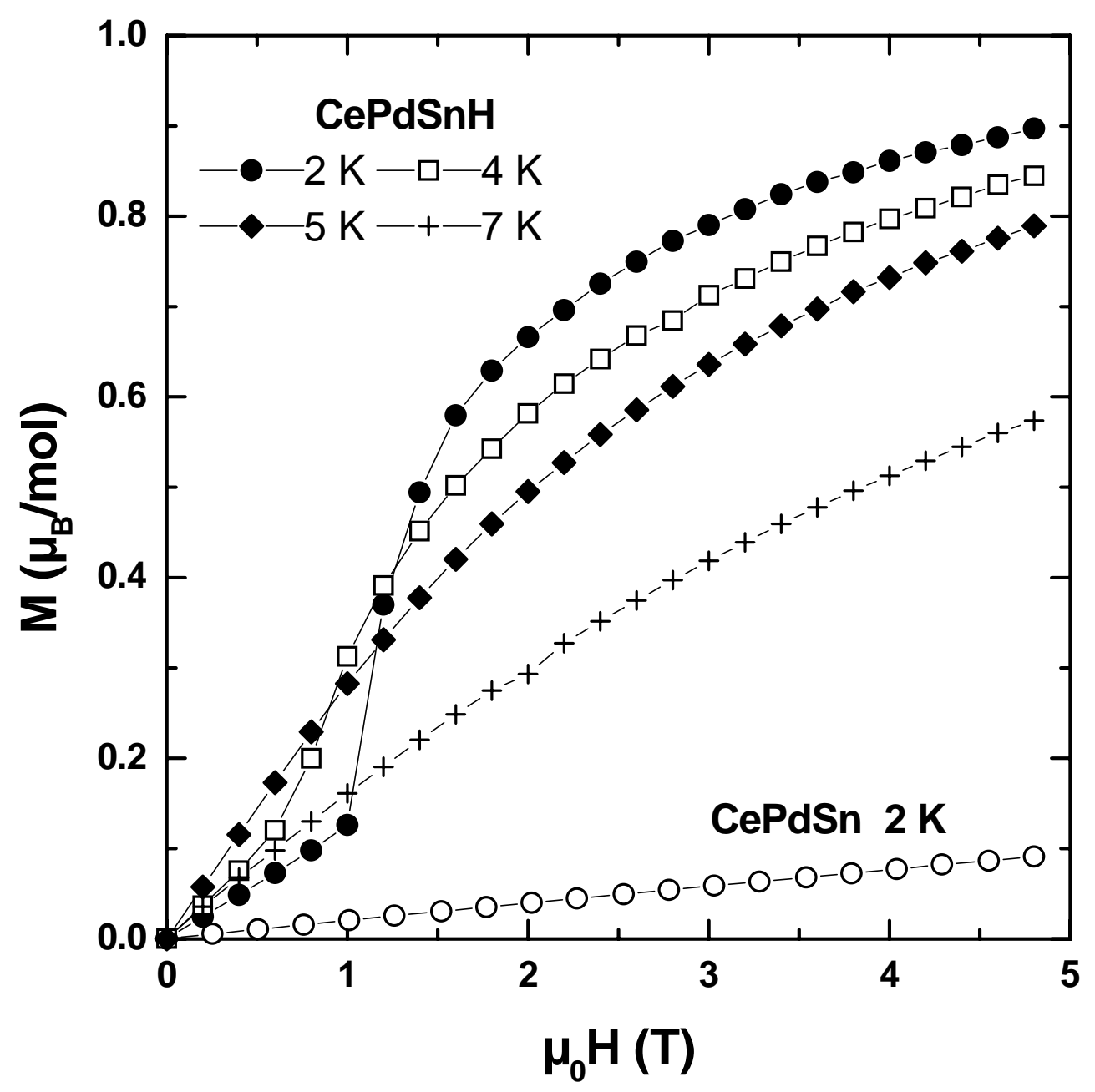

Fig. 10 Field dependence of the magnetization of CePdSn at $2 \mathrm{~K}$ and its hydride at temperatures between $7 \mathrm{~K}$ and $2 \mathrm{~K}$. 


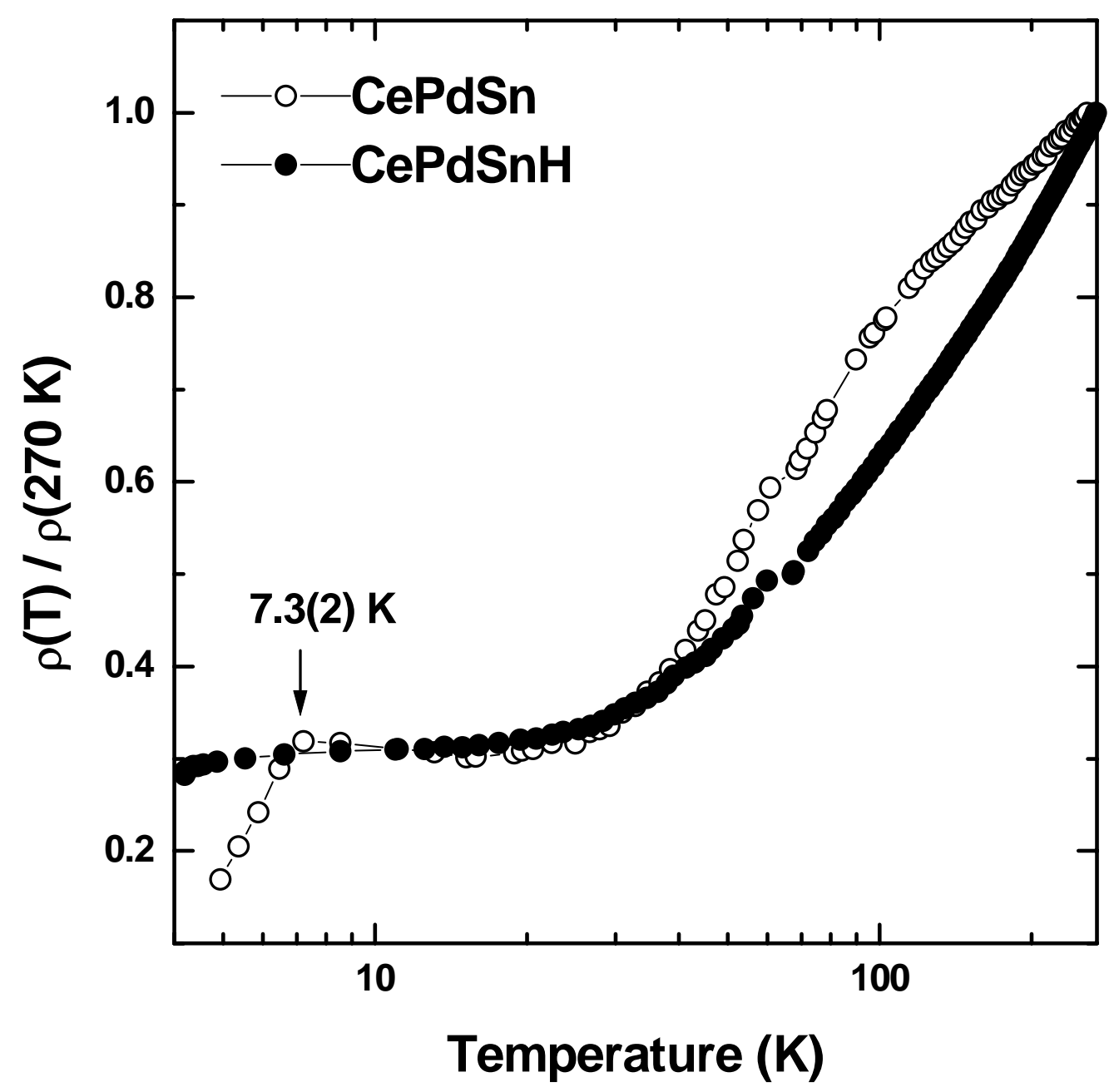

Fig. 11 Reduced electrical resistivity as a function of log T for CePdSn and its hydride. 


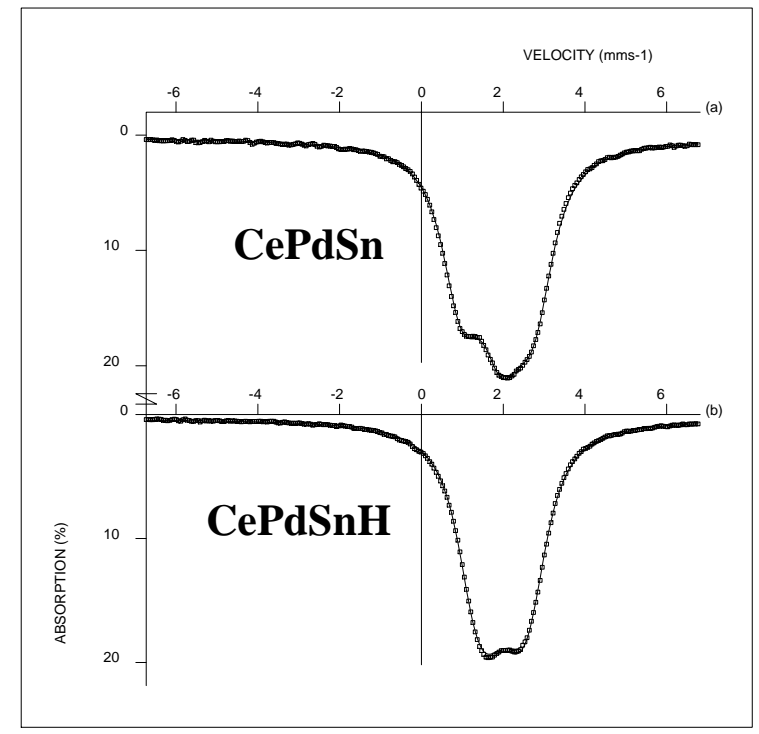

Fig. $12{ }^{119}$ Sn Mössbauer absorption spectra of CePdSn and CePdSnH measured at 4.2 K. The circles represent experimental values, while the lines are fits to the data. 